\title{
A Control Strategy of the Distributed Power Supply to Reduce the Loop Closing Current
}

\author{
Ping Wang, Litao YU, Qiang Wang \\ State Grid Qingdao Power Supply Company, Qingdao, China \\ alyjtxb@163.com
}

Keywords: closing loop current; distributed power supply; control strategy; distribution network. Abstract. In order to improve the reliability of distribution network operation, this paper proposes a method that through adjusting the distributed power output to reduce the closing loop current. Mainly by regulating the operation state of the distributed power to change the power flow distribution, reduce the voltage difference on the ends of the close loop point, thus to reduce the closing loop current value, ensure safe and reliable operation of the power distribution network. Through the simulation example, the method can effectively reduce the closing loop current, has certain guiding significance to the actual distribution network operation scheduling.

\section{Introduction}

Generally speaking, in China the distribution network is radial power supply network, adopts a "closed loop design, open loop operation" mode of power supply. In the maintenance or failure situation, we can achieve uninterrupted load through opening or closing loop, so as to improve power supply reliability. Due to voltage difference exists at the ends of the closing loop point, the closing loop current can be produced when closing the loop. Closing loop operation not only can produce a steady circulation current in the network, and will have a larger impact current on closing loop instant, which will directly affect the safe operation of power grid.

With the development of smart grid, distributed power/energy storage/controllable load content constantly increase in distribution network. It provides a new control method for the dispatching operation of distribution network.

By adjusting the running state of the distributed power supply, we can effectively adjust power flow, reduce the difference voltage on both ends of the closing loop point, thus to reduce the closing loop current. Thus, it's necessary to study the producing mechanism of closing loop current, and develop a control strategy for the distributed power supply that can reduce the closing loop current.

\section{Discussion of production mechanism of closing loop current}

Under normal operating conditions, distribution network structure in China is a radial power supply network. And the schematic diagram of closing loop is as shown in Figure 1. 


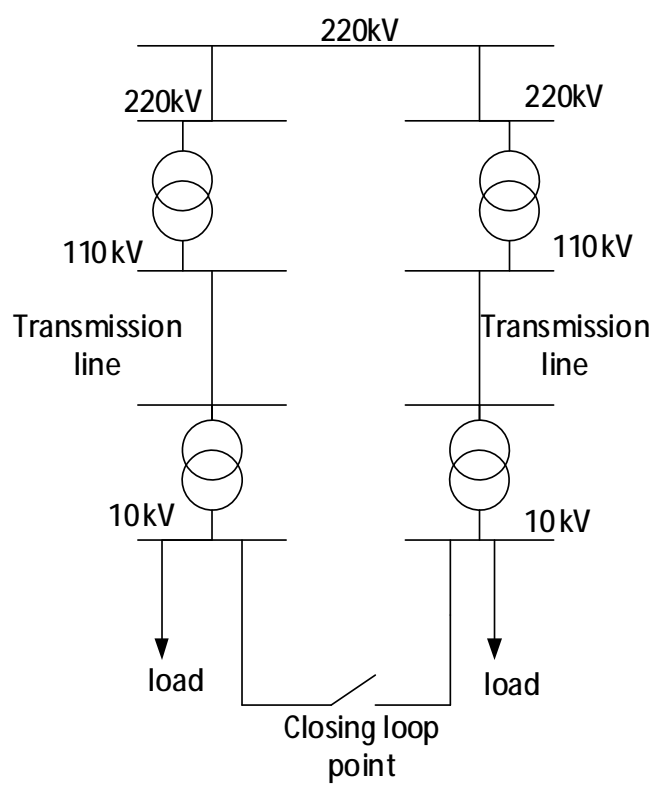

Figure 1 Schematic diagram of closing loop

Assume that a, b stand for the ends of closing loop. Based on Thevenin's theorem, look in to the net from $a$ and $b$, an equivalent circuit as Figure 2 can be formed.

Zeq

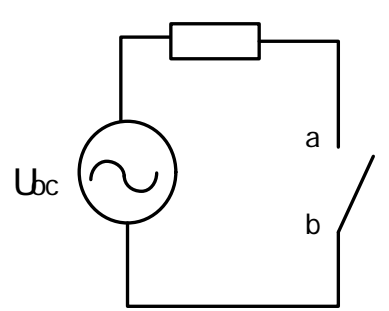

Figure 2 The Thevenin equivalent circuit

In Figure 2, $\hat{U}_{\infty o}$ stands for the equivalent potential, that is the difference voltage between a and $\mathrm{b}$ before the loop is closed; ${ }^{Z}$ stands for the equivalent impedance, that is, for the network of all power supply voltage is zero, the total impedance if we look into the net from $\mathrm{a}$ and $\mathrm{b}$ two points.

Assume that there is a unit current injection in a, $I_{a}=1 \angle 0^{\circ}$, and there is a negative unit current injection in $\mathrm{b}, I_{b}=-1 \angle 0^{\circ}$, and there is no current injection in other nodes. In this way, we can get the voltage value on a and $\mathrm{b}$, and through $U_{a}, U_{b}$, we can get the equivalent impedance $Z_{e q}$.

$$
Z_{e q}=\ddot{U}_{a}-\ddot{U}_{b}
$$

From the node admittance matrix $Y_{n \times n}$, we can get the node impendence matrix $Z_{m \times n}$, and $\mathrm{Z}_{n \times n}=\mathrm{Y}^{-1}{ }_{n \times \mathrm{n}}$. According to $\mathrm{U}=\mathrm{Z}^{\mathbb{1}}$, we can get:

$$
\left(\begin{array}{c}
\dot{U}_{1} \\
\vdots \\
\dot{U} \\
\vdots \\
\dot{U}_{b} \\
\vdots \\
\dot{U}_{n}
\end{array}\right)=\left[\begin{array}{ccccccc}
Z_{1,1} & \cdots & Z_{1, \mathrm{a}} & \cdots & Z_{1, b} & \cdots & Z_{1, \mathrm{n}} \\
\vdots & \vdots & \vdots & \vdots & \vdots & & \vdots \\
Z_{a, 1} & \cdots & Z_{a, \mathrm{a}} & \cdots & Z_{a, b} & \cdots & Z_{a, n} \\
\vdots & \vdots & \vdots & \vdots & \vdots & \vdots & \vdots \\
Z_{b, 1} & \cdots & Z_{b, \mathrm{a}} & \cdots & Z_{b, b} & \cdots & Z_{b, \mathrm{n}} \\
\vdots & \vdots & \vdots & \vdots & \vdots & \vdots & \vdots \\
Z_{n, 1} & \cdots & Z_{n, \mathrm{a}} & \cdots & Z_{n, b} & \cdots & Z_{n, \mathrm{n}}
\end{array}\right]\left(\begin{array}{c}
0 \\
\vdots \\
1 \\
\vdots \\
-1 \\
\vdots \\
0
\end{array}\right)
$$

We can get from the formula that:

$$
\tilde{U}_{a}=Z_{a, a}-Z_{a, b}, \dot{U}_{b}=Z_{b, a}-Z_{b, b}
$$

In the formula, ${ }^{Z_{a, a}}$ is the self impendence of $\mathrm{a},{ }^{Z_{b, b}}$ is the self impendence of $\mathrm{b},{ }^{{ }_{a}, b}$ and ${ }^{Z_{b, a}}$ is the mutual impendence between a and $b$. From formula(1) and formula(3) we can get:

$$
Z_{e q,}=Z_{a, a}+Z_{b, b}-Z_{a, b}-Z_{b, a}
$$


Then we can get the maximum value and the effective value of the instantaneous impact current:

$i_{i m}=\sqrt{2} I_{c}\left[1+\exp \left(-0.01 / T_{a}\right)\right]$

$i_{i m}=I_{c} \sqrt{1+2\left[\exp \left(-0.01 / T_{a}\right)\right]^{2}}$

In the formula $I_{c}=\frac{v_{s x}}{z_{s q}}$ is the steady circulation current after closing the loop switch, $T_{a z}=\frac{\Sigma}{R}$.

So, the impulse current is steady circulation by a fixed coefficient. And both the steady state circulation current and the impulse current is proportional to the difference voltage between the ends of closing loop.

\section{The control strategy of distributed power supply}

Generally speaking we can get the value of active power, reactive power and the amplitude of the voltage from the SCADA system. But, usually, we can't get the voltage phase. Someone put forward that we can get the voltage phase by calculating the power flow of the whole net. But it need a lot of calculations.

The $220 \mathrm{kV}$ and above system is always a ring network with less impendence than the network of the distribute network. So in this paper, we assume that the phase of the $220 \mathrm{kV}$ is zero. In this paper, we calculate the voltage phase by forward and backward substitution method. We only count on the elements that connect the ends of the closing loop to the $220 \mathrm{kV}$ system. And the remaining part of the network equal to loads.

The calculate formulas of forward and backward substitution method is as following:

$$
\begin{aligned}
& \Delta \mathrm{U}=\frac{P_{2} R+Q_{2} X}{U_{2}} \\
& \delta \mathrm{U}=\frac{P_{2} X-Q_{2} R}{U_{2}} \\
& \delta=\tan ^{-1} \frac{\mathrm{dU}}{\mathrm{U}_{2}+\Delta \mathrm{U}} \\
& U_{1}=\sqrt{\left(\mathrm{U}_{2}+\Delta \mathrm{U}\right)^{2}+(\delta \mathrm{U})^{2}}
\end{aligned}
$$

In these formulas, $P_{2}$ is the active power of the ending node, $Q_{2}$ is the reactive power of the beginning node, $\mathrm{R}$ is the resistance of the elements, $\mathrm{X}$ is the reactance of the elements, $\Delta \mathrm{U}$ is the vertical component of the voltage, $\delta \mathrm{U}$ is the horizontal component of the voltage, $U_{1}$ is the voltage of the beginning node, $U_{2}$ is the voltage of the ending node.

In the power system, the active power associates with the voltage phase, the reactive power associates with the voltage amplitude. We can adjust the reactive power by adjusting the inverters of the distributed power supply, and adjusting the controllable loads. We can adjust the active power by collaborative scheduling the distributed power, energy storage, controllable load.

Firstly, we should calculate voltage by the method mentioned above. Then calculate the closing loop current through the method put forward in part 2.If the closing loop current is too large for the system. We can adjust the voltage phase ahead by reducing the active power consumption and increasing the active power output, in the opposite way, we can adjust the voltage phase backward. In a similar way, we can adjust the voltage amplitude greater by increasing the reactive power output and decrease it by reducing the reactive power. In this way, we can reduce the difference voltage between the ends of the closing loop. Then, calculation of the current of closing loop. If the current is still too large for the system, repeat the steps above until the current meets the requirement.

\section{Simulation}

This paper selects the system in Hebi. The structure of the system is shown in Figure 3. 


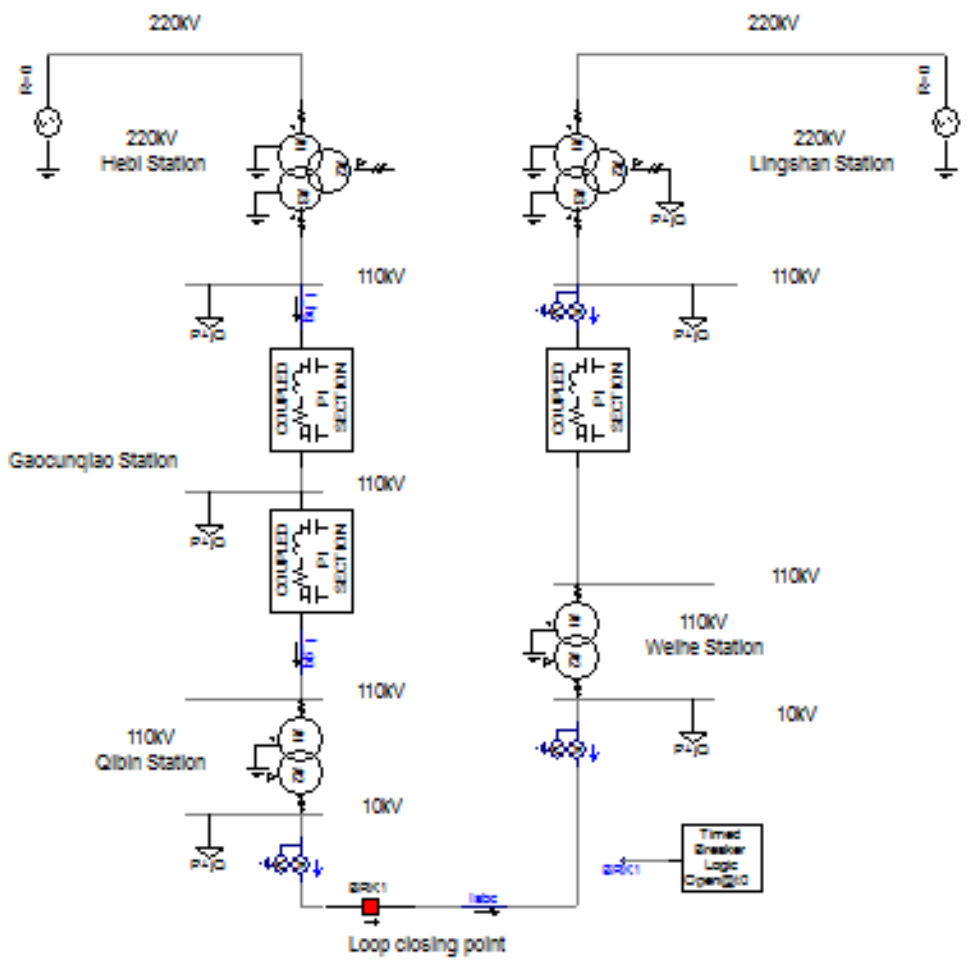

Figure 3 The structure of the power system in Hebi

Assume that there is some controllable distribute power supply content. In the original system, the load of the Qibin station is 9MW, 3MVar; the load of the Weihe station is 4MW, 1.2MVar. When closing the loop, the peak current is 750.7A. After adjustment, the load of the Qibin station is 8MW, $3 \mathrm{MVar}$; the load of the Weihe station is $5 \mathrm{MW}, 1.7 \mathrm{MVar}$. When closing the loop, the peak current is 373A.
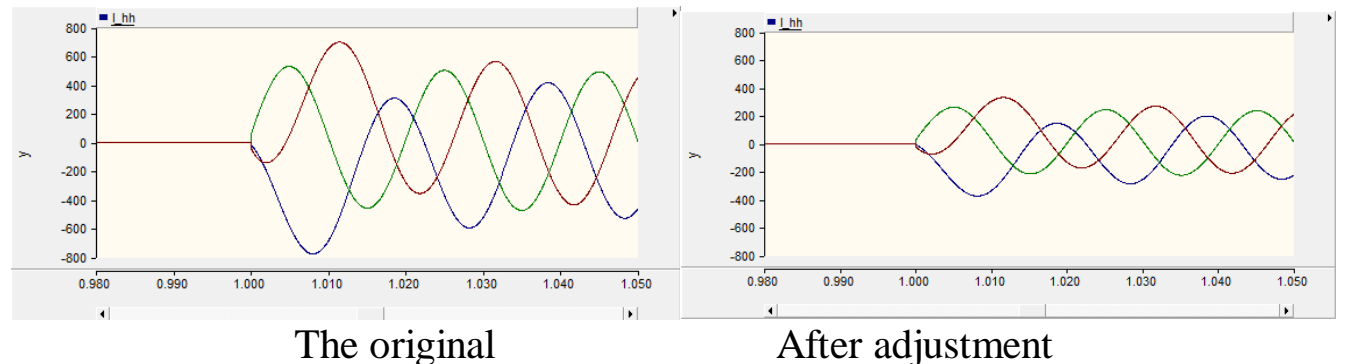

Figure 4 The current of the closing loop

\section{Summary}

This paper first analyses the calculation method of closing loop current, and put forward a control strategy of the distributed power output to decrease the closing loop current. Then through simulation, proved that it's effective to reduce the closing loop current by the control strategy put forward in this paper.

\section{References}

[1] Ge Shaoyun, Li Xiaoming. Calculation of distribution network closing loop impact current based on Thevenin equivalent [J] Proceedings of the EPSA, 2007,19 ( 6 ) : $124 \sim 127$.

[2] Ye Qinghua, Tang Guoqing, Wang Lei, etal. Development and application of the analysis system for closed loop operation of distribution network[J]. Automation of Electric Power Systems, 2002, 26(22): 66-69.

[3] Chen Xiao,W ang Lei, LiYang. Analysis of surge current due to closing loop in distribution grid) [J]. Electric Power Automation Equipment, 2005, 25(4): 40-42. 